\title{
La línea de investigación un referente de especialización en la Escuela de Estudios de Postgrado FAHUSAC
}

\author{
The line of research a reference of \\ specialization in the School of Postgraduate \\ Studies FAHUSAC
}

María Iliana Cardona Monroy ${ }^{1}$

Maribel Valenzuela ${ }^{2}$

Patricia Mazariegos ${ }^{3}$

Walda Flores Luin ${ }^{4}$

Bidel Méndez ${ }^{5}$

https://doi.org/10.46954/librosfahusac.3

\section{Resumen}

OBJETIVO: establecer la perspectiva del profesor, asesor y estudiantes de la Escuela de Estudios de Postgrado de la FAHUSAC en torno a la línea de investigación y su vinculación con una de ellas como fuente de aprendizaje y saber colectivo. MÉTODO: se desarrolló un estudio con enfoque mixto de alcance exploratorio, se aplicó una encuesta a docentes, estudiantes y graduados, además de realizar un análisis documental. RESULTADOS: desde la perspectiva que tienen los informantes se evidencia que existe un posicionamiento

1 Escuela de Estudios de Postgrado, Universidad de San Carlos de Guatemala, Ilicar55@fahusac.edu.gt, https://orcid.org/0000-0003-2749-4605 2 Escuela de Estudios de Postgrado, Universidad de San Carlos de Guatemala, mvalenzuela@fahusac.edu.gt https://orcid.org/0000-0002-6124-9112 3 Escuela de Estudios de Postgrado, Universidad de San Carlos de Guatemala, pmazariegos@fahusac.edu.gt

4 Escuela de Estudios de Postgrado, Universidad de San Carlos de Guatemala, wflores@fahusac.edu.gt. https://orcid.org/0000-0003-0889-1509 5 Escuela de Estudios de Postgrado, Universidad de San Carlos de Guatemala, iih.fahusac@gmail.com 
en torno a las líneas de investigación, se realizan acciones que permiten a los estudiantes apropiarse de la utilidad y la necesidad de contar con estas para realizar investigación, ya que a partir de ellas se produce conocimiento alrededor de los problemas prioritarios que están en el marco de dichas líneas. CONCLUSIÓN: desde la perspectiva del profesor, asesor y estudiantes en torno a las líneas de investigación se conciben como fuente de especialización, y organización, más allá del conocimiento que se produce en torno a ellas, es un potencial teórico metodológico en la investigación y un medio para compartir colectivamente saberes y aprendizajes.

Palabras clave: perspectiva, línea de investigación, vinculación, aprendizaje, saber colectivo, grupos especializados.

\section{Abstract:}

OBJETIVE: establish the perspective of the professor, of the student of the School of Postgraduate Studies of the FAHUSAC around the line of research and its connection with one of them as a source of learning and collective knowledge. METHOD: a study was developed with a mixed approach of exploratory scope, a survey was applied to teachers, students, teachers with pensum closure and graduates, in addition to conducting a documentary analysis. RESULT: from the perspective of the informants, it is evident that there is a position around the research line, actions are carried out that allow students to appropriate the utility and the need to have them to carry out research, since from them knowledge is produced around the priority problems that are within the framework of these lines. CONCLUSION: from the perspective of the professor, advisor and students around the research line, it is conceived as a source of specialization, and organization, beyond the knowledge that is produced around it, is a theoretical and methodological potential in research, in addition to become a means to share knowledge and learning collectively.

Keywords: perspective, line of research, linking, learning, collective knowledge, specialized groups. 


\section{Introducción}

En el contexto educativo la investigación le permite a una institución, proyectarse a nivel social, aportando conocimiento para la mejora de la calidad de vida de una sociedad determinada. En el nivel de educación superior las líneas de investigación han adquirido relevancia porque alrededor de ellas se priorizan problemas para ser investigados y producir conocimiento que permite identificar a profundidad el estado del fenómeno que puede tener diferentes intenciones, siendo una de ellas proponer mejoras.

En el sentido anterior las líneas de investigación han cobrado relevancia en la educación superior. Las Universidades han organizado grupos académicos alrededor de las mismas, estos se conciben como semilleros en la producción de conocimiento. Al respecto se tienen estudios como el presentado por Sanabria y Bonilla (2020) en el cual se caracterizan las líneas estableciendo a los grupos de académicos como unidades de operativización de estas. En la misma línea esta operativización no se trabaja en solitario, no es tarea de un solo investigador, debido a la importancia de la organización académica en torno a las mismas.

En Universidades de Colombia, la organización en torno a las líneas de investigación es bastante avanzada, entre estas destaca la experiencia de la Fundación Universitaria Católica Lumen Gentium (2017), cuyo nivel de organización en torno al tema está bien estructurado, de manera que existen coordinadores de líneas de investigación, lo cual indica que estas son vistas como temas prioritarios para el desarrollo de procesos investigación y la producción de conocimiento. En el sentido anterior Piñon (2020) indica lo importante que es contar con líderes científicos, enfatizando que las líneas son un buen referente para organizarlos.

De manera general se ha encontrado en la líteratura que las líneas de investigación se han desarrollado articuladas en su mayoría a grupos de investigadores, los cuales están 
integrados por docentes y estudiantes y a veces por personal de otras instituciones que desarrollan investigación lo cual es oportuno traer a la vista de esta investigación porque es un medio para fortalecer la multidisciplinariedad, la interdisciplinariedad y la transdisciplinariedad, lo cual es sumamente valioso.

En el caso de la Escuela de Estudios de Postgrado de la Facultad de Humanidades, de la Universidad de San Carlos de Guatemala, en cada uno de los programas que oferta a la población académica nacional como internacional, se produce conocimiento teniendo como referente las líneas de investigación. Estas integran los problemas surgidos de las necesidades de la sociedad y en torno a las cuáles gira el proceso de investigación científica.

Las líneas se conciben como temas prioritarios y genéricos que los estudiantes de los diferentes programas tienen a disposición para realizar su proceso de investigación. Se comprende que los profesores y asesores juegan un papel importante en este sentido, porque coadyuvan a partir de su expertiz, campo disciplinar y posicionamiento en torno a una o varias líneas de investigación a darle significado, a seleccionarla y potenciarla desde las aulas, lo cual es valioso porque el estudiante comprende la importancia y utilidad de estas y no se perciben como un accesorio.

En ese orden de ideas, las líneas de investigación han cobrado relevancia en el contexto universitario, lo cual llevo a la realización de este estudio, con el propósito de establecer el posicionamiento de profesores, asesores y estudiantes en torno a la línea de investigación, que permita dar pasos hacía la organización de grupos académicos.

El interés científico y social para realizar este estudio se enmarca en el hecho de que la educación superior tiene como misión formar investigadores, quienes están llamados a organizarse, lo cual conlleva nuevas funciones investigativas y el desarrollo de otras competencias en los docentes y 
asesores, a fin de potenciar las líneas donde están insertos tanto teórica como metodológicamente, incidiendo en mejores prácticas en torno a la tarea investigativa.

\section{Fundamentación teórica}

A continuación, se incluyen una descripción de la línea de investigación Evaluación Educativa debido a que el estudio se enmarco en esta, además de presentar algunos concepto y teoría sobre el tema de las líneas de investigación.

\section{Línea de investigación evaluación educativa}

Está línea comprende los problemas y temas diversos que tienen que ver con la institución educativa, tales como el control, medición, evaluación y verificación del alcance de los objetivos y fines de la educación, con el propósito de rendir cuentas y develar la calidad de los procesos que se realizan a partir de identificar debilidades, fortalezas y el nivel de logro para encontrar las oportunidades de mejora.

De acuerdo con González y Ayarza (1991) (citado por Mora 2004), la evaluación educativa es una herramienta de mejora, porque permite saber como esta la actividad académica para facilitar la innovación, lo cual es relevante porque incide en la mejora continua de las instituciones, evitando la obsolescencia, de tal manera que se alcancen los objetivos, la misión y la visión institucional.

Esta línea puede enmarcarse en las diferentes funciones de la evaluación educativa, de acuerdo con Elola y Toranzos (2000) puede ser:

a. Política porque sirve de soporte para los procesos de toma de decisiones, no es solo generar conocimiento, sino incidir políticamente para que los cambios se lleven a cabo. 
b. Conocimiento porque describe el fenómeno, proporciona información para la comprensión del problema o la situación que vive la institución.

c. Mejoramiento es complementaria con la función que se enmarca en el inciso a y $b$, dado que la evaluación educativa debe llevar a las instituciones al mejoramiento continúo a partir del conocimiento que se genera y lógicamente en este sentido la incidencia política es fundamental.

En cuanto al alcance, esta línea de investigación comprende diversas temáticas o problemas, contiene a) la evaluación de los aprendizajes, b) evaluación de las instituciones, c) evaluación del sistema educativo, d) evaluación de los programas o proyectos con fines de mejora. En ese marco referencial indicado esta investigación se posiciona en esta línea, dado que los resultados permitirán el fortalecimiento de la actividad investigativa para corresponder a las tendencias del contexto de la educación superior.

\section{La investigación}

La investigación como tal es un proceso asociado a la humanidad a lo largo de la historia en la que se corresponde como resultado de la curiosidad innata del ser humano y de su necesidad de saber, conocer, comprender, interpretar la realidad en que vive, realidad física natural y la social.

Se reconoce, de manera general, que el primer y fundamental producto de la investigación es el conocimiento en todas sus dimensiones, desde el cúmulo de ideas generadas por procesos intelectivos mentales como el proporcionado o encontrado por medio de la experiencia sensorial. 
La naturaleza del conocimiento, y en consecuencia de la investigación, ha generado en todo momento posiciones encontradas en cuanto a su validez. Cada postura, cada planteamiento de identificación o tipificación ha propiciado la determinación de paradigmas o maneras de hacer investigación y así mismo, obtención de variados tipos de conocimiento que, en suma, constituyen el saber humano, su utilización, su buena o mala aplicación. Así, se ha desarrollado la humanidad desde la época de las cavernas a la actualidad expresada como era de la sociedad del conocimiento con el auge evidente de las Tecnologías de la Información y comunicación.

Para orientar o focalizar los procesos de investigación, desde hace algunas décadas se ha promovido determinar líneas de investigación con el propósito de resolver determinadas problemáticas, así como, promover recurso humano que trabaje en forma individual, colectiva, intra e interdisciplinariamente. La USAC a través de la Dirección General de Investigación -DIGI- en cada convocatoria anual estimula la realización de investigaciones presentando una lista amplia de líneas que se enfocan a los grandes temas o áreas de la realidad que corresponden a los diferentes programas universitarios de investigación.

Por su parte, la Facultad de Humanidades, desde inicios de este siglo también ha adoptado la implementación de líneas de investigación, las cuales se han sistematizado desde 2012, cuando cada departamento y escuela de la Facultad determinó sus propias líneas de investigación con la condición que esta se haga con suficiente conocimiento temático (con base al desarrollo de Estados de Arte) y acorde a la naturaleza de los temas/problemas de investigación que se trabajen, es decir, se promueve la realización de estudios con metodologías diversas de carácter cuantitativo, cualitativo o mixto. En ese orden ideas, a continuación, se abordará lo referente a la línea de investigación. 


\subsection{Definiciones de línea de investigación}

Trabajar con base a líneas de investigación, como ya se indicó, es una práctica muy generalizada en la actualidad y resulta interesante que esta forma de orientar la ejecución de estudios investigativos sea concebida de manera diferente según la postura y propósitos institucionales que la promueven. Algunas definiciones con sus particularidades se proporcionan a continuación

Una línea de investigación es un enfoque que abarca conocimientos, inquietudes, prácticas y perspectivas de análisis que permiten el desarrollo de proyectos y productos construidos de manera sistemática alrededor de un tema de estudio. Adicionalmente, concibe el trabajo tanto interdisciplinario como intradisciplinario (Universidad Autónoma de México, s.f.). Se nota que se concibe la línea como un enfoque o sea una forma de ver un tema de estudio entre una disciplina o entre varias. Lo anterior bien podría orientarse a lo que en metodología de la investigación se determina como paradigma o sea una manera específica de hacer investigación.

Por su lado Padrón (1999, párr. 3 y 4) presenta las siguientes ideas al citar a:

Bayley (1995) acoge los términos área-línea, indicando que las líneas se localizan dentro de un área y que ellas son: Niveles de concreción y especifidad que señalan problemas concretos (teóricos y prácticos) cuya necesidad de ser resueltos es evidente y de alguna manera requerida por un sector del entorno (científico, social, educativo, empresarial, entre otros) y para el cual aún sino se tiene todo el personal formado será necesario buscar vías para lograrlo (...) Una línea se plasma en uno o varios proyectos o en un proyecto o fases continuas y tal vez crecientes y progresivas (p.49). 
Resalta en lo citado que la línea es denominación o identificación de un área de la realidad y también, es interesante, el verla con criterio geométrico como sucesión de puntos homogéneos (en este caso investigaciones) con características similares o comunes por su naturaleza y la relación de semejanza y uniformidad. La imagen mental de ver la línea de investigación como línea geométrica es muy ilustrativo cuando se desea trabajar en función de una de ellas, un conjunto de investigaciones, individuales pero continuas que pueden prolongarse infinitamente como el concepto geométrico, a fin de explorar y abarcar lo más ampliamente lo temático que implique.

Para la Escuela de estudios de postgrado de la Facultad de Humanidades de la USAC (2016) la idea de línea de investigación se expresa como “...temas prioritarios y genéricos que permiten ser tratados desde diversos puntos de vista..." (p. 3). En la definición de las líneas de la Escuela se nota una concreción de la idea expresada por Bayley (1995), citado arriba, ya que se determinaron áreas del campo educativo y de la realidad nacional y dentro de ellas un conjunto de líneas propuestas y de las cuales, los investigadores hacen selección para realizar sus estudios de tesis.

Cada línea, en sí, posibilita la realización de una extensa cantidad de estudios acorde a la magnitud de la tematización que se haga dentro de la línea. La referida tematización consiste en listar la mayor cantidad de temas que se puedan determinar dentro de la línea y de esa lista, posteriormente el investigador hace opción por uno acorde a sus intereses y posibilidades y que luego debe profundizar su conocimiento con la realización de un Estado de Arte que le permita documentarse ampliamente y obtener una posición de experto en dicho tema.

Lo descrito posibilita proponer y realizar investigaciones con conocimiento de causa. La ejecución de cada estudio se tipificará metodológicamente acorde al enfoque, diseño y alcance que se determine en cada caso acorde a la naturaleza 
del fenómeno o problema objeto de estudio. En ocasiones, al determinar los temas dentro de la línea de investigación se da la situación que existen subtemas que constituyen una mayor especificación del fenómeno a estudiar y esto daría lugar a considerar a los temas iniciales como sub líneas de la línea en que se generaron.

Para finalizar este apartado es propicio mencionar que, en el medio académico de la USAC y en consecuencia en la FAHUSAC y Escuela de Postgrado a las líneas de investigación se les considera también como "grandes temas" o "macrotemas" en los cuales se pueden denominar muchos temas específicos y que tema es "todo de lo que se puede decir o hablar algo".

\subsubsection{Las líneas de investigación y los intereses institucionales}

Es evidente que cuando una institución determina que para sus procesos de investigación se ha de partir de líneas establecidas, estas representan o expresan los intereses institucionales para los cuales se espera el trabajo de generar conocimiento.

El interés puede surgir de la naturaleza de la misma institución expresada en su visión, misión y objetivos que requieren su logro y que fundamenten la razón de ser de dicha institución.

Así, por ejemplo, la USAC tiene como uno de sus fines contribuir a la solución de los problemas nacionales y esto se aplica a todas sus dependencias (unidades académicas) que deben asumir lo correspondiente en sus acciones particulares. Es decir, trabajar en aquello que contribuya al logro del fin universitario desde la condición, naturaleza y propósito de cada unidad.

Debe quedar claro que los intereses institucionales han de estar concretamente establecidos en las políticas y estrategias que correspondan, en este caso, al campo de la investigación. 
Por ser interesante en este apartado se comparten las ideas de Cerda (2004):

las líneas de investigación... deben necesariamente hacer(sic) parte de un plan de desarrollo donde estén claramente justificadas conceptual, metodológica y técnicamente, y estén consignados sus objetivos, recursos, capitales de interés y todos aquellos aspectos que contribuyan a llevarlas a la práctica: asesorías, cursos de extensión, publicaciones, etc. (. . .) Para muchas universidades las líneas de investigación se constituyen en verdaderas estrategias en la construcción de saberes, de metodología, de procesos de innovación o de creación inter o transdisciplinarios. (p.21)

\subsubsection{Las líneas de investigación y los cuerpos académicos}

Un cuerpo académico es un grupo de profesores que trabaja conjunta y cooperativamente en una disciplina o varias disciplinas. Es un trabajo en equipo en el que se desarrollan habilidades y aptitudes docentes e investigativas que conducen a productos y acciones que contribuyen a la caracterización y eficiencia de los programas educativos, así mismo, el trabajar en grupo, permite el confrontamiento de posiciones y saberes que enriquecen a sus participantes que a su vez se consolidan en una especialidad.

Para ser parte efectiva de un cuerpo académico ha de privar un amplio concepto de tolerancia y de desprendimiento para poder aceptar los planteamientos y posiciones de los pares, así como tener la facilidad de compartir con los otros lo que personalmente se sabe, se trabaja, se elabora. Es una relación de entrega y recepción sin egoísmos que promueve formación profesional en un ambiente sereno y serio de análisis y comprensión. 
El que una institución educativa promueva sus procesos de investigación con base a líneas de investigación le posibilita integrar Cuerpos Académicos que permitan desarrollar estudios cada vez más efectivos por cuanto los docentes al trabajar en torno a una temática determinada se van haciendo expertos en ella y en consecuencia sus acciones y productos serán cada vez de mayor calidad.

Como es normal, en todo programa educativo (carrera), actúa un grupo de profesores que se desempeñan en disciplinas diversas según el diseño del programa y por lo tanto al integrar un Cuerpo Académico en torno a una línea de investigación estarán dando el plus, en busca de la interdisciplinariedad que posibilita la diversidad de visiones y tratamientos integrales de una problemática específica. La calidad del servicio educativo se ve incrementada y cimentada cuando hay un efectivo trabajo de Cuerpos Académicos y los beneficios de esto se manifiestan en los docentes y estudiantes con saberes ricos y fundamentados.

Estratégicamente, la institución educativa con Cuerpos Académicos ha de promover la rotación periódica de los integrantes de dichos cuerpos, que, si bien se potencia la especialización, también el dejarlos permanentemente en un mismo grupo conducirá indefectiblemente a la obsolescencia de sus miembros. Todo cambio implica un reto y el cambiar de Cuerpos Académicos en determinados tiempos enfrenta a los docentes a ser mejores en otras áreas y esto constituye prestigio y crecimiento personal e institucional.

\subsubsection{Las líneas de investigación y la asesoría educativa}

Es práctica generalizada que cuando un estudiante de pregrado, grado o postgrado realiza su trabajo de graduación traducido en una investigación científica asociada a una línea de investigación, es acompañado de un tutor o asesor que se encarga de asistirle, de apoyarle en el desarrollo de su estudio. Ese apoyo puede traducirse en acciones como correcciones, 
sugerencias, ilustraciones, emisión de opiniones dentro del entorno y desarrollo que implique el proyecto de investigación, es, un proceso de cogestión en el que asesor contribuye a la concreción efectiva de la investigación dentro de los parámetros institucionales.

En términos educativos, el asesor de investigación realiza un proceso de asesoría educativa que encaja en lo que la Fundación Universitaria María Cano (2007, p. 5) define así:

Asesoría es orientación al estudiante en el proceso de aprendizaje en el desarrollo como persona, como profesional y como miembro de la comunidad. (...) Es de tipo investigativo, según la necesidad específica de cada estudiante de acuerdo con su proyecto de investigación, el asesor trabajará con el alumno, enfatizando en la línea de investigación correspondiente (...) poniendo en el centro del quehacer formativo, el desarrollo integral de la persona $(\mathrm{s} / \mathrm{p})$.

La cita suficientemente expone la importancia que tiene el asesor en el desempeño y logro del estudiante ya que el acompañamiento que se dé no solo estriba en desarrollar un producto académico sino implica el crecimiento de la persona del estudiante.

En investigación, el trabajo del asesor que en la mayoría de los casos es con investigadores nóveles, inexpertos, en crecimiento profesional, es de suma importancia por cuanto de sus consejos $u$ orientaciones se estará gestando la carta de presentación del futuro profesional que se le ha encomendado, tarea digna y a toda honra de un educador, en este caso, un educador investigativo. 


\subsection{Los beneficios de trabajar con líneas de investigación}

En lo desarrollado hasta aquí, es notoria la importancia que tiene que se trabaje con líneas de investigación ya que esto implica hacerlo dentro de las expectativas del país manifiestas en las demandas que implican las áreas y líneas que se promueven para contribuir al desarrollo científico y tecnológico, esto desde las estructuras estatales establecidas para ese fin, como también lo que significan en el quehacer de las instituciones que se dedican a la investigación y dentro de estas, las universidades que se constituyen en centros obligados de realizar investigación para cumplir con sus fines y las demandas de la sociedad.

Como colofón de esta exposición se presentan las ideas de González y Núñez de la Universidad de Pinar del Río "Hermanos Saiz Montes de Oca", Cuba, que en su artículo "Conceptualización y definición de líneas de investigación prioritarias a nivel de la Universidad" exponen lo que en Ecuador se consideran como los principales beneficios del establecimiento de LIP para las universidades. (en la cita se encuentran las siguientes siglas: LIP = Líneas de Investigación Prioritarias; $\mathrm{CE}=$ Centros de Estudio, $\mathrm{GI}=$ Grupos de Investigación)

- Estimulan el trabajo coordinado y cooperativo entre investigadores y estudiantes que pertenecen a diferentes áreas administrativas de la universidad alrededor de los CE o GI que lideran las LIP, propiciando el fortalecimiento de estos y de los resultados que se obtengan.

- Permiten articulación de proyectos de investigación que están dispersos en una misma área del conocimiento y en diferentes áreas administrativas de la propia institución. 
- Identifican de forma clara la trayectoria productiva de un conjunto de investigadores, proyectos de investigación, CE y GI.

- Permiten la asignación y uso más racional de los recursos destinados a la investigación en la universidad.

- Las LIP permiten a los profesores y estudiantes planear y orientar su trabajo investigativo dentro de una visión amplia, pero al mismo tiempo precisa.

- Garantizan que la formación doctoral tribute de manera directa a la solución de problemas de elevada pertinencia y relevancia social.

- El ejercicio de pensar la universidad en función de las problemáticas nacionales y territoriales, permite ubicar el desempeño pasado, presente y futuro de las mismas ante las interrogantes y complejidades científicas, tecnológicas, culturales, sociales, políticas y económicas que deben ser resueltas con el aporte de su comunidad.

- Para la formación de pregrado supone basar el desempeño de los docentes en el ejercicio del método científico, orientando a los estudiantes en sus prácticas laborales investigativas a la búsqueda de soluciones en el marco de su perfil profesional como agentes activos en la construcción del nuevo conocimiento. Así como también el perfeccionamiento de los programas de estudio en función de las LIP.

- Permiten evaluar el desempeño científico de la universidad en su conjunto a partir de los resultados alcanzados en cada una de las LIP al final de cada periodo de planificación en términos de resultados académicos, científicos, tecnológicos, relevancia e impactos (González y Núñez, 2020, párr. 27). 


\section{Materiales y métodos}

El estudio se sustenta epistemológicamente en la perspectiva teórica del pensamiento complejo, que aborda la realidad desde una visión multidimensional, considera la realidad cambiante, incierta, reconoce los fenómenos en conexión con otros, no los considera aislados de su contexto. De acuerdo con Morin (citado por Zenteno y Osorno 2014), "toda ciencia debe considerar la unidad, diversidad, continuidad y las rupturas" (p. 32), así mismo se sustenta en el paradigma hermenéutico interpretativo. Esta investigación tuvo un enfoque mixto, con alcance exploratorio, el método cuantitativo tuvo más énfasis que el cualitativo. Fue un estudio transeccional, se recolectó información en un solo momento, a partir de técnicas e instrumentos diseñadas para el efecto.

El diseño fue triangulación concurrente, de manera simultanea se recolectaron datos cuantitativos y cualitativos a partir de la aplicación de un cuestionario que incluyó una serie de preguntas cerradas con su propia escala de respuesta, en coherencia con los objetivos de la investigación, dirigido a profesores y asesores de la Escuela de Estudios de Postgrado y un cuestionario dirigido a estudiantes y graduados diseñado con preguntas abiertas. Se realizó análisis documental para establecer las líneas de investigación que se han seleccionado de manera recurrente en la Escuela de Estudios de Postgrado. Finalmente se realizó la interpretación y discusión de la información recolectada

La muestra fue seleccionada de forma intencionada, tanto para la fase cuantitativa como para la cualitativa, los criterios de inclusión y exclusión se listan a continuación:

Los criterios de inclusión, profesionales que estén ejerciendo docencia y asesoría en la Escuela de Estudios de Postgrado, profesores y asesores que pertenecen a la EEP, estudiantes de la maestrías categoría ciencias que están asignados en los cursos de Seminario de Tesis, estudiantes que realizaron la defensa de tesis en el 2021. 
Los criterios de exclusión, profesores que no ejercen docencia en las maestrías categoría ciencias, asesores externos, estudiantes de los otros ciclos de las maestrías categoria ciencias, estudiantes que se retiraron del programa.

\section{Resultados y discusión}

Los resultados que a continuación se presentan corresponden al objetivo de la investigación: establecer la perspectiva de profesores, asesores, estudiantes y egresados en torno a la línea de investigación como fuente de aprendizaje y saber colectivo en la Escuela de Estudios de Postgrado.

Los datos descriptivos se obtuvieron tras aplicar instrumentos a 16 profesores y asesores, 16 estudiantes activos de las maestrías en ciencias y doctorado, así como a 75 informantes que comprenden maestrandos con cierre de pensum, que están en las última fase de la realización de tesis y profesionales egresados y estudiantes de las maestrías categoría ciencias que están asignados en los cursos de Seminario de Tesis. Así mismo con el análisis documental se obtuvo un inventario que evidencia las líneas de investigación que se han seleccionado de manera recurrente en la Escuela de Estudios de Postgrado entre los años 2017 a 2021.

Es importante mencionar que la investigación en la educación superior responde a políticas institucionales que como establece Díez (2013); Arcila (1996); Agudelo (2004); Barrera (2000) y Kuznig et al. (2010) se convierten en esfuerzos encaminados a reforzar la investigación, innovación y el desarrollo en beneficio de la sociedad. La Universidad de San Carlos de Guatemala como respaldo a una de sus funciones sustantivas como la investigación, cuenta con su propia política, que precisamente en la actualidad está en revisión a fin de que responda a las demandas y desafios del contexto. 
Así mismo cada unidad académica de la USAC, hace sus propios esfuerzos para impulsar la investigación. En la Facultad de Humanidades especificamente en la Escuela de Estudios de Postgrado en los programas de Maestrías en Ciencias, Maestrías en Artes y Doctorado se cuenta con áreas y líneas de investigación que se han establecido como temas genéricos y prioritarios alrededor de los cuales se han realizado estudios desde diversos paradigmas y enfoques de investigación, aportando conocimiento que refleja perspectivas, prácticas, significados, subjetividades de los fenómenos abordados.

En la actualidad las líneas de investigación, no solo son un referente de sistematización de conocimiento, sino que también de organización del personal académico con el fin de contar con grupos que se convierten en expertos que desde diversos campos disciplinares pueden orientar la producción de conocimiento en la línea o líneas de investigación en la cual se posicionan como expertos.

En ese sentido la línea de investigación ha cobrado relevancia en el contexto académico porque posibilita la realización de una extensa cantidad de estudios acorde a la magnitud de temas que comprenda la línea. El investigador elige uno de esos temas que se encuentra dentro de una línea que responde a sus intereses y expertiz, así como a otras razones tales como la emergente necesidad de proponer soluciones a problemas reales.

Lo anterior también permite organizar a los investigadores o cuerpos académicos alrededor de las líneas de investigación quienes juegan un papel fundamental, ya que van a orientar al aprendiz investigador a seleccionar la línea de investigación sobre la cual versará su estudio, de tal manera que se realizan las investigaciones con conocimiento de causa y no se aborda la línea como un accesorio que solamente se menciona en el estudio, pero no dimensiona la importancia de esta. 
De acuerdo con Sumac et al. (2015); Puerta (2008); Rodríguez (2011) y Tamayo (1999) la línea de investigación, se convierte en una necesidad, lo cual indica la importancia que un docente o asesor de investigación configure su saber alrededor de una determinada línea o varias líneas de acuerdo con su campo disciplinar, establecida en la institución de educación superior. En universidades a nivel latinoamerciano han realizado procesos para organizar la investigacion con el fin de fortalecerla, una de las formas ha sido a través de las líneas de investigación, estableciendo su respectivo grupo de investigadores, lo cual mejora la producción de conocimiento.

En ese sentido en la Escuela de Estudios de Postgrado se realizó este estudio para explorar la perspectiva de los informantes con relación a las líneas de investigación, posicionamiento y su vinculación a una o varias líneas, los resultados que se presentan no suman el $100 \%$ pues las respuestas eran excluyentes debido a que los encuestado podían seleccionar varias respuestas.

Los resultados relevantes evidencian que de los profesores y asesores que imparten docencia en las maestrias en ciencias, el $80 \%$ se vincularon con las líneas de investigación del área investigación educativa aplicada, un $73 \%$ con educación superior, un $47 \%$ problemática educativa nacional, un $40 \%$ con el área social humanista, 33\% con la epistemología de las ciencias sociales y un $20 \%$ con realidad social y política de Guatemala. Mientras que, en el doctorado, el $78 \%$ se vincula al área de investigación y educación, un $67 \%$ aprendizaje, un $44 \%$ a Curriculum y diversidad cultural, un $22 \%$ se vincula a filosofía y teoría de la educación, uso de las Tics en educación y un $11 \%$ a gestión educativa e institucional.

Se evidencia que son 9 líneas de investigación a nivel general de las 33 que existen en el programa de Maestrías en Ciencias y 32 en el programa de Doctorado, con las que se vincula el profesor, sin duda, son las líneas en la cual tiene expertiz y tiene relación con el campo disciplinar. Sin embargo, es importante mencionar que hay líneas que no han 
sido vinculadas o reconocidas por los profesores y asesores como parte de su quehacer investigativo y docente, esto implica muy poca producción de conocimiento alrededor de las mismas, lo cual deja rezagados problemas o temas que institucionalmente han sido priorizados para ser investigados, pero que sin embargo no han cobrado mucha relevancia para los actores implicados.

Los resultados anteriores se ratifican cuando se indaga acerca de las líneas que han sido seleccionadas de manera recurrente para hacer investigación por parte de los maestrandos con cierre de pensum y egresados, se evidenció que las líneas: calidad del sistema educativo, educación y trabajo, evaluación educativa y curriculum están contenidas en el área problemática educativa nacional donde se posicionaron el $47 \%$ de los profesores encuestados, así mismo la línea de investigación: didáctica de la educación superior se encuentra en el área de educación superior donde se posicionaron el $73 \%$ de los profesores y finalmente se tiene la línea de tecnología educativa que pertenece al área de investigación educativa apliacada donde se posicionaron el $80 \%$ de los profesores. Lo anterior lleva a pensar que los profesores de alguna manera con el proceso educativo que desarrollan y el acompañamiento que dan a los estudiantes dejan aprendizajes y experiencias que los ha llevado a elegir esas líneas donde se encuentra el expertiz de los docentes $y$ asesores. 


\section{Tabla 1}

Líneas de investigación seleccionadas de manera recurrente para realizar investigación

\begin{tabular}{|c|c|c|}
\hline Resumen por líneas asumidas & $\mathbf{F}$ & $\%$ \\
\hline Didáctica de la educación superior & 14 & 18.67 \\
\hline Calidad del sistema educativo & 10 & 13.33 \\
\hline Tecnología educativa virtual & 6 & 8.00 \\
\hline Curriculum & 6 & 8.00 \\
\hline Educación y trabajo & 6 & 8.00 \\
\hline Evaluación educativa & 7 & 9.35 \\
\hline $\begin{array}{l}\text { Educación superior, realidad nacional y des. } \\
\text { Hum }\end{array}$ & 5 & 6.67 \\
\hline Modalidades educativa & 3 & 4.00 \\
\hline Deserción y repitencia & 2 & 2.67 \\
\hline Educación en salud & 2 & 2.67 \\
\hline Estudios de género & 3 & 4.00 \\
\hline Tecnología de la información y redes sociales & 2 & 2.67 \\
\hline Asentamientos humanos & 1 & 1.33 \\
\hline Educación y género & 1 & 1.33 \\
\hline Epistemología de la investigación & 1 & 1.33 \\
\hline La educación superior en la globalización & 1 & 1.33 \\
\hline Políticas educativas & 1 & 1.33 \\
\hline Seguridad alimentaria y salud & 1 & 1.33 \\
\hline Entornos virtuales & 1 & 1.33 \\
\hline Estrategias de aprendizaje & 1 & 1.33 \\
\hline Modalidades educativas & 1 & 1.33 \\
\hline total & 75 & 100.00 \\
\hline
\end{tabular}

Fuente: propia.

Este resultado se torna en una línea base acerca de la producción científica en torno a las líneas de investigación.

De acuerdo con el trabajo realizado por González y Nuñez (2020), las líneas articulan saberes provenientes de distintos campos disciplinares estimulando el trabajo colaborativo, cooperativo y coordinado entre investigadores y estudiante, tal es el caso de las universidades. En ese sentido es necesario agrupar a los profesores y asesores de acuerdo a expertiz 
y experiencia a las líneas que han identificado, también es oportuno articular profesores y estudiantes alrededor de esas líneas de investigación que han pasado desapercibidas, según los autores referidos, lo más díficil de contar con líneas de investigación es operativizarlas, y acreditar conocimiento alrededor de ellas, ya que contar con muchas líneas de investigación puede constituirse en una limitante.

Lo anterior debe motivar la revisión de las líneas existentes con el fin de promover investigación en aquellas líneas que cuentan con poca producción de conocimiento sobre los problemas y temas que contiene, estas tienen relación con el campo educativo, filosófico, curricular e investigativo, entonces cabe hacer el esfuerzo para operativizar las líneas que se tienen definidas en la institución.

Haciendo alusión a la percepción de estudiantes, indican que la línea de investigación da sentido a los estudios, permiten priorizar los problemas, ubican la problemática en un área de estudio, son una fuente para fomentar el trabajo interdisiciplinario, siendos estos puntos de encuentro con la percepción de los profesores quienes también indicaron que la línea de investigación es una fuente para conformar grupos de trabajo y fortalecer la interdisciplinariedad en la investigación, así mismo la definieron como una base racional de la actividad investigativa, dado que ayudan a ordenar el conocimiento que se produce alrededor de las mismas. (ver tabla 2 y 3) 


\section{Tabla 2}

Percepción de estudiantes en torno a la línea de investigación

\begin{tabular}{lc}
\hline \multicolumn{1}{c}{ Expresiones } & $\mathbf{f}$ \\
\hline $\begin{array}{l}\text { Ayuda a teorizar } \\
\text { Dar a conocer las líneas de investigación con más frecuencia }\end{array}$ & $2 / 16$ \\
$\begin{array}{l}\text { Comprender las líneas brinda un panorama para futuros proyectos } \\
\text { de investigación. }\end{array}$ & $3 / 16$ \\
$\begin{array}{l}\text { En lo personal considero que es una forma de mantener el camino } \\
\text { correcto al seguir la investigación y tener claridad que el tema } \\
\text { planteado está correcto }\end{array}$ & $1 / 16$ \\
$\begin{array}{l}\text { Definir las líneas de investigación en la guía para realización de } \\
\text { tesis }\end{array}$ & $1 / 16$ \\
$\begin{array}{l}\text { La línea hace que el estudio sea específico } \\
\text { Base para la investigación y un parteaguas en la formación } \\
\text { académica }\end{array}$ & $3 / 16$ \\
$\begin{array}{l}\text { Comprender las líneas brinda un panorama para futuros proyectos } \\
\text { de investigación. }\end{array}$ & $2 / 16$ \\
\hline
\end{tabular}

Fuente: propia.

Para obtener la percepción de los profesores se les presentaron cuatro opciones de respuesta en la encuesta, los resultados de la tabla 3 presenta la frecuencia con que cada profesor seleccionó la opción de respuesta.

\section{Tabla 3}

Percepción de profesores en torno a la línea de investigación

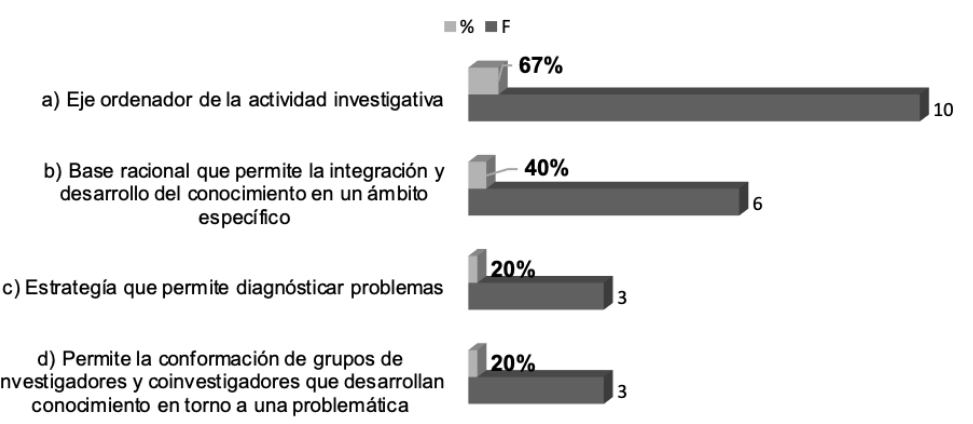


Un proyecto de investigación bajo el enfoque interdisciplinar consistirá en la integración de los resultados de investigación. Pero en este caso y a diferencia de la investigación poli o multidisciplinar, sobre todo en el caso de la generación de nuevas disciplinas, la integración se hace desde el comienzo a partir de un diálogo abierto entre los investigadores y de los aportes que cada investigador y su método pueden brindar para la construcción común del objeto de análisis.

Adicional a esto, al finalizar la investigación, los resultados son validados por cada uno de los miembros del equipo, lo cual lleva en muchos casos a una superación de los esquemas cognitivos con los que opera una determinada disciplina. Por tanto, los resultados de investigación pueden expresarse a través de una novedosa y creativa interpretación de la realidad estudiada o mediante la construcción de un nuevo esquema cognitivo: la interdisciplinariedad (Osorio, 2012, p. 284).

Con relación a lo anterior, en la Escuela de Estudios de Postgrado los profesores podrían aplicar un enfoque interdisciplinar para realizar investigación en torno a la línea en la cual se posicionan, podrían constituirse en productores de conocimiento de la linea de su especialidad, desde las diferentes miradas de acuerdo con el campo disciplinar de los grupos que integren.

Finalmente se determinó con la información recolectada desde la perspectiva de los profesores encuestados que realizan diversas actividades para promover la aplicación de la línea de investigación con la que se vincula entre las cuales se tienen: teorizar sobre las líneas de investigación en el aula, generar espacios de discusión en torno a la línea de investigación, presentación de temas generadores y como temas introductorios al inicio de los cursos. Desde la perspectiva del asesor se da acompañamiento, se brindan técnicas para seleccionar la línea y se proporciona referencias bibliográficas para orientar al estudiante investigador al elegirla, y desarrollarla. 
Los estudiantes indican que los profesores promueven la aplicación de las líneas de investigación desde la temática de los cursos, dando seguimiento a la guía para elaboración de los planes y los informes de investigación, en este aspecto coinciden las versiones de los estudiantes con la de los profesores.

Lo anterior lleva a reflexionar en torno al trabajo que se realiza en la Escuela de Estudios de Postgrado con relación a la producción de conocimiento alrededor de las líneas de investigación, es importante establecer que la calidad de la educación que se brinda, se fortalece a partir del trabajo que realizan los profesores con sus estudiantes, por lo que es importante la organización estratégica de los académicos en torno a las líneas de investigación, lo cual permitirá la operativización de la mayor parte de las líneas de investigación, a partir de la conformación de un grupo de profesores.

\section{Conclusiones}

La perspectiva de los docentes y asesores sobre la línea de investigación es diversa, es importante mencionar que los docentes de las maestrías en ciencias la mayoría $80 \%$ se vinculan con líneas del área de investigación educativa aplicada, en el doctorado el mayor porcentaje $78 \%$ se vincula con el área de investigación y educación. Uno de los significados de la línea de investigación para estos informantes es que contribuye a generar aprendizajes y saberes colectivos con los estudiantes.

Se logro establecer que los docentes se posicionan como expertos en algunas de las líneas que incluye la Escuela de Postgrado en sus diferentes programas, además de que, en su función de asesores, ese posicionamiento ha permitido que puedan acompañar adecuadamente a los estudiantes en la correcta selección y aplicación de la línea de investigación en sus trabajos investigativos según comentarios de los propios estudiantes. 
Se identificó la línea de investigación con la cual se vincula el profesor y asesor de la Escuela de Estudios de postgrado en su quehacer profesional, esto permitió evidenciar que de las 33 líneas de investigación disponibles solamente se utiliza de forma recurrente el $27 \%$, esto es un indicativo de que se deben organizar grupos de docentes en torno a todas las líneas establecidas para poder realizar acciones que permitan aumentar la aplicación del 100\% de las líneas de investigación tanto en la Maestría como en el doctorado.

Se determinó que el docente en su función de docente y de asesor promueve la aplicación de las líneas de investigación a través de procesos de socialización de la o las líneas en que tiene expertiz, orientando a los estudiantes sobre generalidades de estas y su vinculación con el trabajo de investigación que los estudiantes realizan.

Se estableció que son 21 las líneas de investigación que seleccionan de manera recurrente los estudiantes al realizar el trabajo de investigación para egresar del programa que cursa, seleccionando con mayor frecuencia las líneas de Didáctica de la Educación Superior y Calidad del sistema educativo, esto evidencia la necesidad de revisar cuidadosamente las líneas que se seleccionan con menos frecuencias para promocionarlas adecuadamente a manera de que sean visibles y que el estudiante tenga la opción de identificar si se alinean o no a su estudio. 


\section{Referencias}

Arcila, O. (1996). Las líneas de investigación como elemento articulador deprocesos académicos en la Universidad, Universidad central de Bogota, Colombia. Revista Nómada. No. 5 https://www.redalyc.org/ pdf/1051/105118998013.pdf

Agudelo, N. (2004). Las líneas de investigación y la formación de investigadores: una mirada procesos formativos. Revista ieRed: Revista Electrónica de la Red de Investigación Educativa [en línea]. Vol.1 (1) http:// revista.iered.org.

Barrera, J. (2000). Metodología de la investigación holística. Instittuto Universitario de tecnología. Caripito. SYPAL.

Cerda, H. (2004). Hacia la construcción de una línea de investigación. Seminario Taller. Facultad de Educación. CIFE. Colección Investigación. Editorial Universidad Cooperativa de Colombia.https://books.google. com>books>about>Hacia_la_c...

Díez, L. (2013). Los sistemas de investigación en los países europeos desentralizados. Estudio comparado de España, Alemania, Austria y Bélgica, y de Cataluña, Babiera, Estiria y las regiones belgas. Cataluña,

España. Institut d'Estudis autonómics.

Elola, N. y Toranzos, L. (2000). Evaluación educativa: una aproximación conceptual. Biblioteca digital Universidad Academia Humanismo Cristiano. http://bibliotecadigital. academia.cl/xmlui/handle/123456789/585

Facultad de Humanidades, Escuela de Estudios de Postgrado (2016). Guía para la elaboración del plan de investigación, presentación del informe final y líneas de investigación de tesis. Guatemala. s.m.d.e.

Fundación Uiversitaria Católica Lumen Gentium (2017). Acta de Reunion - grupo de investigación interdisciplinario en conocimiento organizacional - GICO- https://repository.unicatolica.edu. co/bitstream/handle/20.500.12237/1364/

Acta\%2 OGIICO\%20Diciembre\%204-2017\%20. pdf?sequence $=1$ \&isAllowed $=y$ 
Fundación Universitaria María Cano. (2007). Manual del asesor educativo. $2^{\mathrm{a}}$. Edición. Rocío Builes Bedoya, compiladora. Medillin, Colombia. FUMC.

https://www.fumc.edu.co $>$ resoluciones $>a r c \_3528$

González, M. y Nuñez, S. (2020). Conceptualización y definición de líneas de investigación prioritarias a nivel de la universidad. Revista Universidad y Sociedad Volumen 12(4).

Kuznik, A. Hurtado, A. y Espinal, A. (2010). El uso de la encuesta del tipo social en traductología, características metodológícas. Universidad autónoma de Barcelona, España. Monografías de Traducción e Interpretación. No. 2 www.Redylac.org/artículo

Mora, V. (2004). Evaluación Educativa: conceptos, periodos y modelos. Revista electrónica: Actualidades Investigativas en Educación. 4(2) https://www.redalyc. org/pdf/447/44740211.pdf

Osorio, G. (2012). Pensamiento complejo y la transdisciplinariedad: fenómenos emergentes de una nueva racionalidad. Vol. XX (1). http://www.scielo.org. co/pdf/rfce/v20n1/v20n1a16.pdf

Padrón, G. (1999). El concepto de línea de investigación y sus implicaciones. Caracas. UNESR.

Piñon, J. (2020). El líder científico en la universidad pedagógica de nuestros tiempos. Revista Cubana de Educación Superior. 39(3). http://scielo.sld.cu/scielo.php?script=sci_abstract\&pid=S0257- 43142020000300006\&ln$\mathrm{g}=$ es\&nrm=iso

Puertas, M. (2008). Diseño de líneas de investigación en las Instituciones Universitarias. Restrepo, B. (2003). Investigación http://www.indexf.com/lascasas/ documentos/lc0300.php

Rodríguez, A. (2011). Líneas de investigación y dialogismo en los procesos de investigativos en el campo Universitario ecuatoriano. Revista de Estudios Latinoamericanos. No. 54. 
Sanabria, N y Bonilla, L. (2020). Caracterización de las líneas de investigación. Acta académica. http://revista. uaca.ac.cr/index.php/actas/article/view/905/1212

Sumac, S. Posso, M. y Caicedo, F. (2015). Línea de investigación: importancia para las instituciones de educación superior. Ecos de la Academía.

Tamayo, M. (1999). El proyecto de investigación. Serie Aprender a Investigar. ICFES. Colombia.

Universidad Autónoma de México (s.f). Áreas de conocimiento y líneas de investigación. División de investigación. Facultad de Contaduría y Administración.http://investigacion.fca.unam.mx/ docs/folleto/folleto_DIFCA.pdf

Zenteno, B. y Osorno, S. (2014). Elementos para el diseño de investigaciones jurídicas. Una perspectiva multidimensional. Universidad de Puebla México. Piso 15 editories. https://archivos.juridicas.unam.mx/ www/bjv/libros/9/4301/10.pdf

\section{Semblanza académica de los autores}

\section{Ph. D. María Iliana Cardona Monroy}

Doctora en Investigación, Directora de la Escuela de Estudios de Postgrado, asesora del curso de Seminario a nivel de grado, asesora de tesis a nivel de Maestría y Doctorado, frecuentemente escribe artículos científicos en revistas indexadas.

\section{Ph. D. Maribel Alejandrina Valenzuela Guzmán}

Doctora en Educación por la Universidad de San Carlos de Guatemala. Coordinadora del Programa de Maestrías en Ciencias. Docente asesora, revisora y evaluadora de Tesis en Maestrías y docente del curso Seminario de tesis I en el Doctorado en Innovación y Tecnología Educativa en la Escuela de Estudios de Postgrado. Revisora de las revistas científicas de la escuela de Postgrado FAHUSAC. 


\section{Ph. D. Patricia Luz Mazariegos Romero}

Doctora en Educación con especialización en investigación por la Universidad Internacional Iberoamericana -UNINIMéxico, beca FUNIBER. Coordinadora del Doctorado en Innovación y Tecnología Educativa, asesora y revisora de tesis de Maestrías y Doctorado en la Escuela de Estudios de Postgrado. Revisora par profesional de la Revista Científica del Sistema de Estudios de Postgrado -SEP- Revisora de las revistas científicas de la escuela de Postgrado FAHUSAC.

\section{Ph. D. Walda Paola Flores Luin}

Doctora en educación con énfasis en Mediación Pedagógica por la Universidad La Salle de Costa Rica. Docente, asesora, revisora y evaluadora de tesis en la Escuela de Estudios de Postgrado.

\section{Maestro José Bidel Méndez}

Maestro de educación primaria urbana, profesor de enseñanza media en Pedagogía, Letras y Ciencias con especialidad en Química, Licenciado en Pedagogía y Ciencias de la Educación, Maestro en investigación. Actualmente Director del Instituto de Investigaciones Humanísticas de la Facultad de Humanidades, USAC. Asesor de EPS en carreras de grado. Asesor, revisor y evaluador de tesis en la Escuela de Estudios de Postgrado. 